\title{
Postal ban proposed on exchange of dangerous microbes
}

Washington

RESPONDING to pressure brought by Jeremy Rifkin and his Foundation on Economic Trends, the US Postal Service is proposing to ban sending diseasecausing microbial agents and toxins through the mail. The ban would mean that some 100 microbes that are now exchanged between research institutions

\section{NASA panel seeks more life in space}

\section{Washington}

President Reagan's call for a permanent US presence in space has rekindled commitment to space exploration and made particularly timely a new report* by a National Aeronautics and Space Administration (NASA) advisory committee. The Life Sciences Strategic Planning Study Committee (LSSPSC) emphasizes the importance of life sciences to the success of future manned space flights.

The report contends that the success of life sciences depends on quick and easy access to space, something a space station should provide. A space station would also permit long-term experiments to fill gaps in current knowledge about human survival in outer space.

To broaden the expanse of NASA's knowledge in sustained space operations, the committee also recommends cooperating with the Soviet Union on experiments, as well as encouraging universities to participate more actively in NASA's programme.

The LSSPSC report complements an earlier report ${ }^{\dagger}$ by the NASA Center Science Assessment Team (CSAT) which singled out life sciences as one of two speciality areas of science on which NASA should expend more time and effort. The CSAT report contends that a shortcoming of the NASA programme was a lack of long-term goals in the life sciences division. The LSSPSC report attempts to rectify that.

Although there is a fairly large body of data on the effects of prolonged spaceflight on human physiology and biochemistry, these data have not been well integrated into a theoretical framework. The LSSPSC report also indicates a need to pursue exobiological experimentation. A better understanding of the origin of life on this planet could well come from the exploration of how life evolved, or failed to evolve, on other planets.

Lisa L. Lyles

*Exploring the living universe: a strategy for space life science. A report of the NASA Life Sciences Strategic Planning Study Committee. Washington, DC 1988.

$\dagger$ Science at the NASA field centers. A report of the NASA center science assessment team. Washington, DC, 1988. under special packaging requirements will have to be sent by other means.

The Assistant Postmaster General last week announced the new policy at a Congressional hearing on the dangers of sending biological materials through the mail.

Regulations have been on the books since 1980 to ensure that dangerous microbes - such as the bacterium which causes anthrax, and the Crimean Congo haemorrhagic fever virus - are shipped in proper packaging. Rules set down by the US Department of Health and Human Services (HHS) currently require that vials containing such microbes be placed into three successively larger containers along with enough packing material to absorb the culture if the vial should break. The outermost container must carry a universal biohazard symbol and a hotline number at the US Centers for Disease Control (CDC). The most dangerous cultures must be sent by registered mail so that the sender receives confirmation that the package has arrived safely. The Department of Transportation also has authority over the interstate shipping of hazardous cultures of over $50 \mathrm{ml}$.

The ban proposed by the postal service would cover the specific list compiled by HHS of some 100 microbes that are known to cause human disease. Some are responsible for dreaded diseases such as Ebola fever or plague, but others are routinely exchanged in many areas of scientific research. All species of mycoplasma and mycobacteria are on the list, as are hepatitis and herpesviruses, simian virus 40 , and all serotypes of Escherichia coli which can cause intestinal disorders.

The CDC and the American Type Culture Collection (ATCC), the US repository for all isolated microbial strains and genetic material, are the two most frequent mailers of biologically hazardous materials. CDC mailed about 1,000 disease-causing cultures over the past 17 months, and the ATCC reportedly shipped 900. Ft Detrick, Maryland, the centre of the Army's biological defence programme, claims to have sent only 48 cultures of hazardous microbes through overnight private courier services.

\section{DFG re-elects Markl}

\section{Munich}

THE members of the Deutsche Forschungsgemeinschaft (DFG) re-elected president Hubert Markl almost unanimously to a second three-year term last week at the DFG's annual meeting in Bonn. The term runs from January 1989 to December 1991.

Steven Dickman
Network for Europe

\section{London}

THE European network for research on the neural mechanisms of learning and memory has got off the ground with the first meeting of the coordinating committee. The network, proposed at the European Science Foundation general assembly last November, aims to provide a general forum where European workers in this research area can discuss work in progress with the goal of encouraging collaborative and interdisciplinary research projects.

European research in learning and memory has been suffering from poor exchange of information and ideas in a field that uses techniques ranging from biochemistry to animal learning and computer modelling. As a first move to fill this gap, the committee will set up a register of laboratories working in the field and organize several small specialist workshops. The initial phase of the network, lasting two years, has a budget of FFr 560,000 to fund these workshops and one open meeting, which will be held in the spring of 1990 , probably in London. The committee will also promote links between experimentalists and modellers through liaison with programmes, such as the artificial-intelligence-based BRAIN project.

The emphasis will be on several wellestablished experimental preparations, to prevent too broad a spread of resources, but regular discussion meetings will ensure that new developments are not overlooked and results are seen in a wider context. If the first phase is successful, the network will be extended, and suitable projects may receive twinning grants.

Information about the network can be obtained from the secretary, Professor Jean Delacour, Université de Paris VII, Laboratoire de Psychophysiologie, 7 quai St Bernard, Bât.B, 5,ét., F-75251 Paris Cedex 05, France.

Jennifer Altman

The CDC report that no problems have been encountered with the current policy governing the shipment of diseasecausing microbes. The CDC hotline averages only 50 calls per year reporting damaged packages with biohazard labels, and only three per year turn out to be actually leaking. No one has so far developed disease from a leaking package.

But what most concerns Congress is that no federal agency now monitors whether or not the packaging rules for disease-causing microbes are being followed regularly. At the Congressional hearing last week, a postal employee from the district in Maryland which handles mail to and from Ft Detrick reported several instances where improperly packed cultures opened during normal handling: one was even alarmingly marked "retrovirus". 